\title{
Single Incision Approach for Decompression of Greater, Lesser and Third Occipital Nerves in Migraine Surgery
}

\section{Danielle Olla}

Southern Illinois University School of Medicine

Brian Mailey ( $\sim$ brian.a.mailey@gmail.com )

San Diego https://orcid.org/0000-0002-2846-7556

\section{Research article}

Keywords: Migraine Surgery, Occipital Migraines, Greater Occipital Nerve, Lesser Occipital Nerve, Third Occipital Nerve, Single Incision

Posted Date: July 28th, 2021

DOI: https://doi.org/10.21203/rs.3.rs-744343/v1

License: (c) (i) This work is licensed under a Creative Commons Attribution 4.0 International License. Read Full License 


\section{Abstract \\ Background}

The traditional approach for occipital migraine surgery encompasses 3 separate surgical incisions in the posterior neck to decompress the great occipital nerves (GON), lesser occipital nerves (LON) and third occipital nerves (TON). We sought to evaluate a single midline incision approach for decompression of all 6 occipital nerves.

\section{Methods}

Using 10 cadaveric hemi-sides ( 5 fresh cadaver head and necks). Anatomic landmarks and the location of the bilateral GON, LON and TON were marked according to previous anatomic studies. A single midline 9-cm incision was made and lateral skin flaps were raised to decompress or avulse all 6 nerves.

\section{Results}

The GON and TON were identified 3.5 and $6.2 \mathrm{~cm}$, respectively inferior to a line bisecting the external auditory canal (EAC) and $1.5 \mathrm{~cm}$ lateral to the midline. The LON was identified $6-\mathrm{cm}$ inferior and $6.5-\mathrm{cm}$ medial to a line bisecting the EAC in the plane just above the investing layer of the deep cervical fascia until the posterior boarder of the sternocleidomastoid was encountered. The LON had the greatest amount of variation, but was identified lateral to the posterior border of the SCM.

\section{Conclusions}

A single midline incision approach allows for successful identification and decompression of all 6 occipital nerves in migraine surgery.

\section{Background}

Migraine headaches ranked as the third most prevalent disorder in the world in 2010 and the third-highest cause of disability worldwide in both males and females under the age of 50 years in $2015 .{ }^{2}$ The pain of occipital migraines is located in the upper neck and occipital region is associated with stress, muscle tightness, trigger point tenderness, and may be related to heavy exercise or history of whiplash. ${ }^{10-}$

${ }^{11}$ Advances in the underlying pathophysiology have led to promising treatment modalities, such as botulinum toxin A (BTX-A) injections and migraine surgery. ${ }^{7-8}$ Traditional release of these nerves involved in occipital migraines requires 3 separate incisions in the posterior neck.

Anatomic studies have defined the location of each of the nerves associated with occipital migraine headaches. These include the bilateral great occipital nerves (GON), bilateral lesser occipital nerves (LON) 
and bilateral third occipital nerves (TON) ${ }^{12}$ The location of these nerves and their emergence from under muscle and fascia is key for successful treatment with BTX-A injections or surgical treatment. (Table 1) In a systematic review, migraine headache surgery reported an average success rate of $90 \%$ with either elimination or $50 \%$ or greater improvement of migraine headaches. ${ }^{9}$ Sixty-two percent of patients with occipital migraine headaches reported total relief of migraine symptoms and all patients had some element of improvement in migraine headaches after open release of $\mathrm{GON}{ }^{8}$

Table 1

Location of previously defined sites of great occipital (GON), lesser occipital (LON) and third occipital nerves (TON).

\begin{tabular}{|lll|}
\hline & Distance from midline & Distance from line between auditory canals \\
\hline Great occipital nerve & $1.5-\mathrm{cm}$ & $3.0-\mathrm{cm}$ \\
\hline Lesser occipital nerve & $6.5-\mathrm{cm}$ & $5.3-\mathrm{cm}$ \\
\hline Third occipital nerve & $1.3-\mathrm{cm}$ & $6.2-\mathrm{cm}$ \\
\hline
\end{tabular}

Previously, occipital migraine surgery has been described with a $4.0-\mathrm{cm}$ to $4.5-\mathrm{cm}$ midline incision to address the GON and TON. There are then two separate incisions placed laterally near the sternocleidomastoid (SCM) in the lateral neck to address the LON. This leaves the patient with 3 separate incisions increasing the risk of pain, wound breakdown, scarring, neuroma formation and is not ideal of the aesthetic appearance of the posterior neck. In this study, we describe an approach to occipital migraine surgery with a single incision to safely decompress the GON, TON and the more laterally located LON.

\section{Methods}

Ten cadaveric hemi-sides were provided by Southern Illinois University. The cadaveric head was placed in the prone position. The posterior boarder of the SCM was marked bilaterally. A line was then drawn in the horizontal plane at the level of the external auditory canal (EAC) followed by a line vertically down midline from level of occipital protuberance to the base of the neck. The GONs were marked $1.5-\mathrm{cm}$ from the midline and $3.5-\mathrm{cm}$ from the EAC. The LONs were marked $6.5-\mathrm{cm}$ from midline and $6-\mathrm{cm}$ from the EAC. The TONs were marked $1.3-\mathrm{cm}$ from midline and $6.2-\mathrm{cm}$ from the EAC. ${ }^{12}$ We then designed a $9-\mathrm{cm}$ long midline incision in the caudal occipital region down into the superior neck (Fig. 1).

The incision was made through the skin and subcutaneous tissue down to the midline raphe. Large subcutaneous skin flaps were raised laterally just above the investing layer of the deep cervical fascia. When the posterior board of the SCM was encountered, dissection was continued with spreading technique to identify the LON along the posterior boarder of the SCM. Once the LON was identified, it was followed superiorly to confirm its identity and avulsed or sites of compression were released until the nerve entered the subcutaneous tissue. 
The GON and TON were routinely addressed after the LON to avoid confusing the planes. The trapezius fascia was incised $0.5-\mathrm{cm}$ lateral to midline leaving the midline raphe intact. When present, the oblique trapezius muscle was retracted laterally. The semispinalis capitus was found just below the fascia running in the vertical direction and dissection was carried subfascial until trunk of GON was identified. $2.5-\mathrm{cm}$ of the semispinalis muscles medial to nerve was excised. Each compression point was released as the nerve was followed distally to its entrance to the subcutaneous tissue. During the release of the GON, the TON was encountered a similar distance from midline but inferior to the GON. It was avulsed as its sensation contributions are small. (Fig. 2 and Fig. 3)

\section{Results}

Ten cadaveric hemi-sides were dissected. Each of the nerves were sequentially identified. The GON and TON were consistently located at their anatomic landmarks. The LON had more variation in its location and could be challenging to identify in some instances. The most common dissecting error encountered was being in a deeper plane than the SCM. The posterior border of the SCM lies superficial to the subcutaneous tissues of the neck and the LON is easiest to identify emerging posterior to the SCM muscle and coursing upwards towards the occiput. The great auricular nerve (GAN) emerges superior to the LON and courses more anterior. The GAN can be mistaken for the LON, as they both emerge posterior to the SCM. Tracing the nerve to its final location avoids inadvertent injury to an unintended structure, like the GAN.

The LON varies in its caliber from 1-4 mm in size and at its emergence from the SCM can course near the spinal accessory nerve (SAN). The SAN runs obliquely and inferior to the LON, but their emergence around the SCM can be at a similar location. Following the LON to the posterior occiput can confirm its identity. A nerve stimulator can also help confirm if motor fibers are present.

After each nerve was dissected and photos were taken, the skin flaps were retracted to follow the course of the nerves to further confirm their identities. The LON was challenging to locate in 2 ( 4 hemi-sides) of the cadaver heads requiring more extensive and prolonged dissection. Keeping the dissection plan superficial to the SCM helped maintain landmarks and ease the identification of the nerves. The dissection plane created from the midline only requires skin flap elevation of 3-4 additional centimeters on each side and can provide a broader perspective on the location of each nerve.

\section{Discussion}

Traditionally occipital migraine surgery is performed with 3 separate incisions to decompress the GON, TON and LON. More incisions can increase pain, neuroma formation and risk of wound breakdown. Scars on the back tend to widen and many patients are female with concern for aesthetic appearance of the scars. We have successfully performed a single incision release of all 6 nerves in 6 hemi-necks. The consistent and well-defined anatomic location of the GON, LON and TON makes this single incision approach safe and feasible. 
During the dissection it is essential to create large skin flaps to achieve adequate exposure. The skin flaps should be elevated just above fascia to preserve the musculature and fascial planes. The fascia should be entered just at the posterior boarder of the SCM to locate the LON. It is also important to note the course of the LON as it emerges from the posterior aspect of the SCM and then travels on the anterior surface of the SCM. The great auricular nerve emerges just inferior and follows a similar course. ${ }^{16}$ The TON is in close in proximately to the GON located just inferior as it pierces the semispinalis muscle but is smaller in caliber and can be easily missed.

After completing this study, we now perform this technique in occipital migraine surgery. In the preoperative area, we mark the posterior boarder of the SCM with the patient turning head laterally against resistance in the pre-operative area and the precise location of the LON can be identified as the examiner rolls his fingers over the posterior border of the SCM, the location of pain signifies the location of the LON. Decompression of nerves is performed by technique described above. 1 drain is placed under the subcutaneous flaps and secured in place to minimize fluid accumulation and dead space. The incision is closed in a layered fashion.

\section{Conclusions}

Occipital migraine surgery has been established as a beneficial treatment option, but traditionally requires 3 separate incisions to release the paired GON, LON and TON. These six nerves can all be accessed and released through a single midline incision. The course of the LON has the most variation and be the most challenging to locate, however, the wider midline approach can provide anatomic perspective for successful identification of each of the nerves, while preserving the planes and musculature in the posterior neck.

\section{Abbreviations}

GON

greater occipital nerve

LON

lesser occipital nerve

TON

third occipital nerve

EAC

external auditory canal

SAN

spinal accessory nerve

GAN

great auricular nerve

SCM

sternoscleidomastoid muscle 
BTX-A

botulinum toxin $\mathrm{A}$

\section{Declarations}

Availability of data and material: Not applicable

Author contributions: DO and BAM performed cadaver dissections. They were both were major contributors in writing the manuscript. All authors read and approved the final manuscript

Funding: No funding was obtained for this study

Acknowledgements: Not applicable

Competing interests: None

Source of cadaver head and necks: Cadaver head and necks were provided by Southern Illinois University. No permissions or consent that was required for use in your study.

Ethics approval and consent to participate: This article does not contain any studies with human participants or animals performed by any of the authors.

Consent for publication: For this type of study informed consent is not required

The authors declare that they have no conflicts of interest to disclose.

\section{References}

1. Burch RC, Loder S, Loder E, Smitherman TA. The prevalence and burden of migraine and severe headache in the united states: Updated statistics from government health surveillance studies. Headache. 2015;55(1):21-34.

2. Leonardi M, Raggi A. Burden of migraine: International perspectives. Neurol Sci. 2013;34(Suppl 1):117-8.

3. Puledda F, Messina R, Goadsby PJ. An update on migraine: Current understanding and future directions. J Neurol. 2017;264(9):2031-9.

4. Olesen J, Burstein R, Ashina M, Tfelt-Hansen P. Origin of pain in migraine: Evidence for peripheral sensitisation. Lancet Neurol. 2009;8(7):679-90.

5. Penfield W. A contribution to the mechanism of Intracranial Pain. Assoc Res Nerv Dis Proc. 1935; 15:399-416.

6. Ray BS, Wolff HG. Experimental studies on headache. Pain sensitive structures of the head and their significance in headache. Arch Surg. 1940;41:813-56. 
7. Guyuron B, Reed D, Kriegler JS, Davis J, Pashmini N, Amini S. A placebo-controlled surgical trial of the treatment of migraine headaches. Plast Reconstr Surg. 2009;124(2):461-8.

8. Guyuron B, Kriegler JS, Davis J, Amini SB. Comprehensive surgical treatment of migraine headaches. Plast Reconstr Surg. 2005;115(1):1-9.

9. Janis JE, Barker JC, Javadi C, Ducic I, Hagan R, Guyuron B. A review of current evidence in the surgical treatment of migraine headaches. Plast Reconstr Surg. 2014;134(4 Suppl 2):131S-41S.

10. Totonchi A, Guyuron B. Surgical treatment of migraine headaches. In: Losee J, ed. Plastic Surgery: Volume 3: Craniofacial, Head and Neck Surgery and Pediatric Plastic Surgery, Fourth Edition. Canada: Elsevier Inc; 2018: 82-91.

11. Guyuron B, Becker D. Surgical Management of Migraine Headaches. In: Guyuron B et al editor. Aesthetic Plastic Surgery Video Atlas. China: Elsevier Inc; 2012. pp. 313-23.

12. Dash KS, Janis JE, Guyuron B. The lesser and third occipital nerves and migraine headaches. Plast Reconstr Surg. 2005;115(6):1752-8. discussion 1759-60.

13. Mosser SW, Guyuron B, Janis JE, Rohrich RJ. The anatomy of the greater occipital nerve: Implications for the etiology of migraine headaches. Plast Reconstr Surg. 2004;113(2):693-7; discussion 698-700.

14. Janis JE, Hatef DA, Ducic I, et al. The anatomy of the greater occipital nerve: Part II. compression point topography. Plast Reconstr Surg. 2010;126(5):1563-72.

15. Lee M, Brown M, Chepla K, et al. An anatomical study of the lesser occipital nerve and its potential compression points: Implications for surgical treatment of migraine headaches. Plast Reconstr Surg. 2013;132(6):1551-6.

16. Brown DL, Dellon AL. Surgical approach to injuries of the cervical plexus and its peripheral nerve branches. Plast Reconstr Surg. 2018;141(4):1021-5.

\section{Figures}




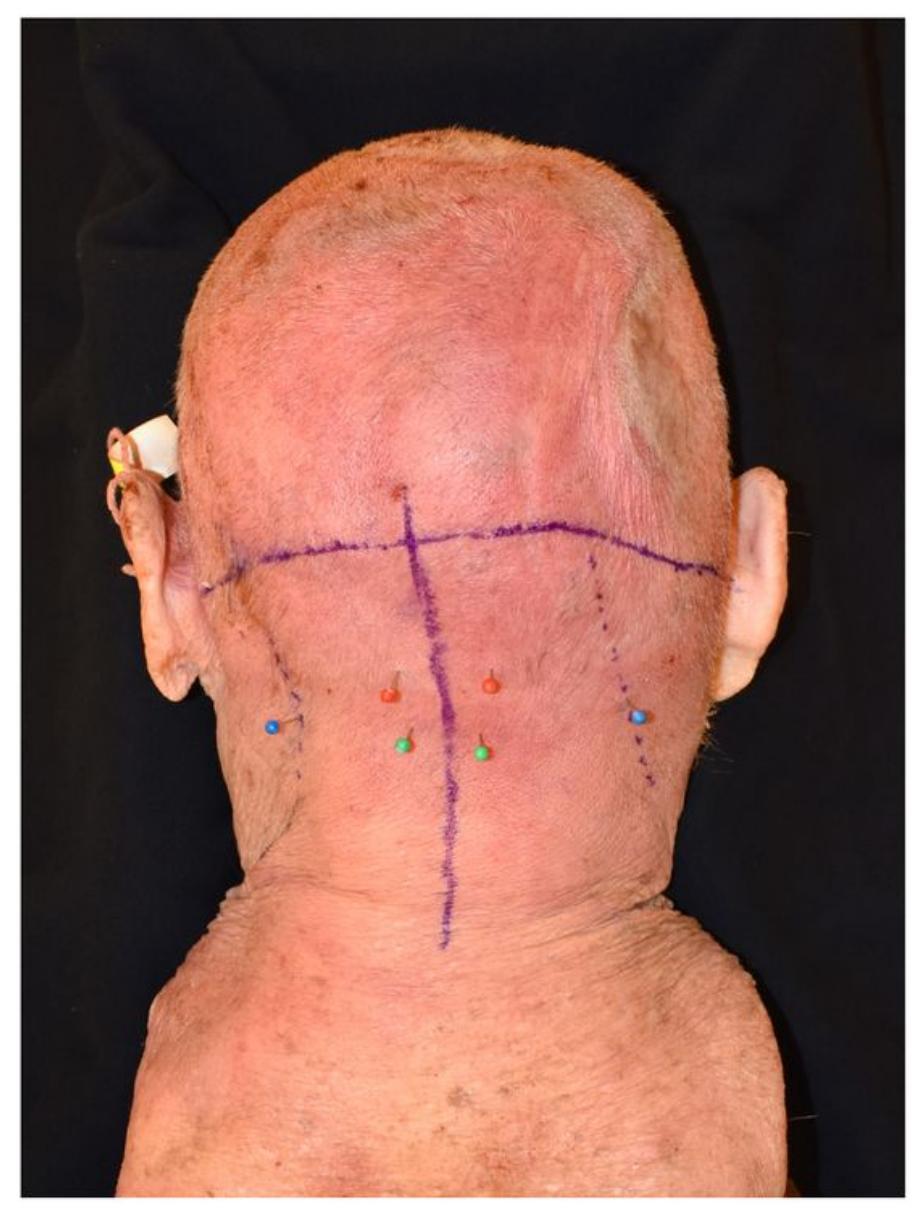

A

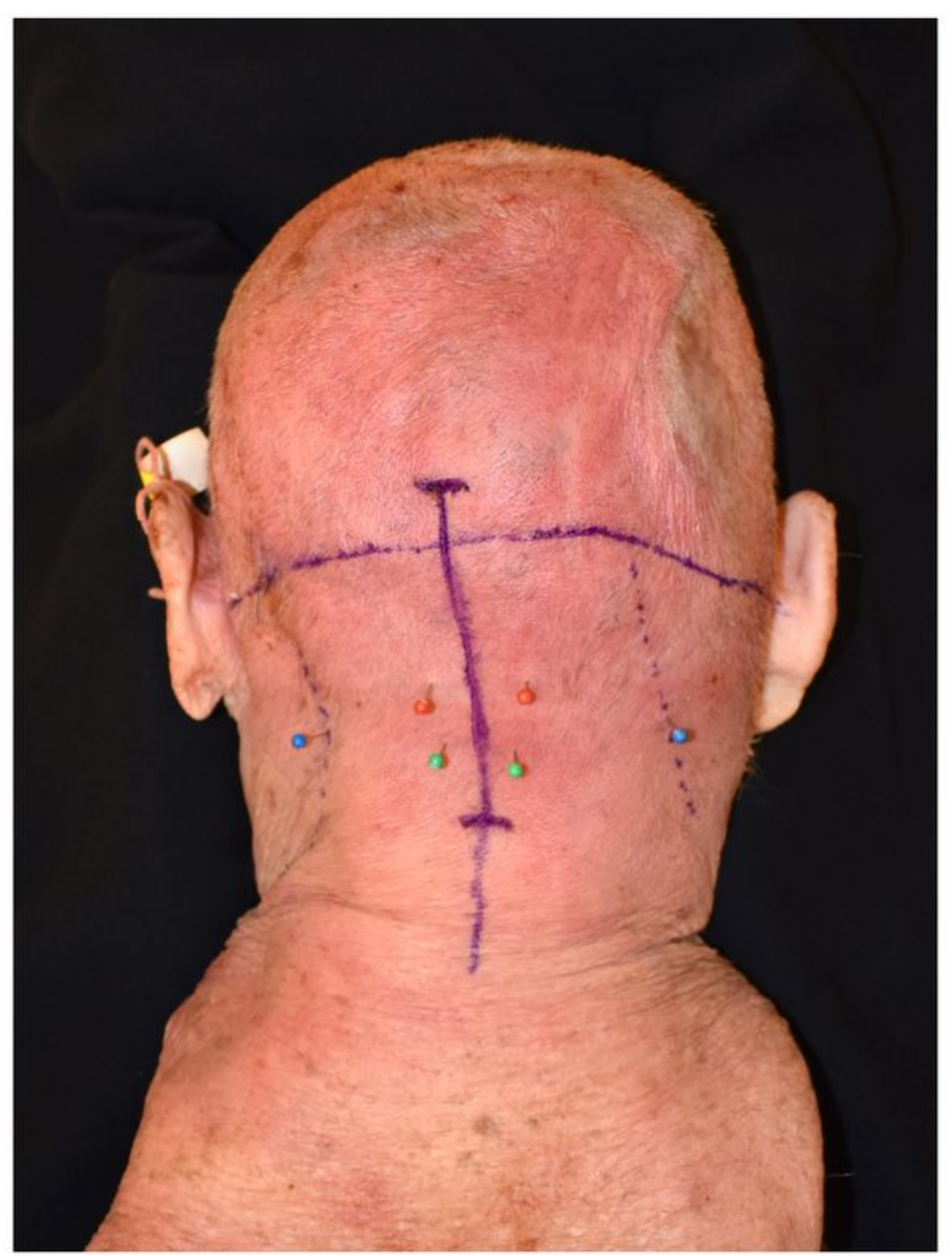

B

\section{Figure 1}

A. Marked anatomic location of GON 1.5-cm from the midline and 3.5-cm from the EAC (red), LON 6.5-cm from midline and $6-\mathrm{cm}$ from the EAC (blue), TON 1.3-cm from midline and 6.2-cm from the EAC (green) B. Marked 8-cm midline incision in the caudal occipital region down into the superior neck. 


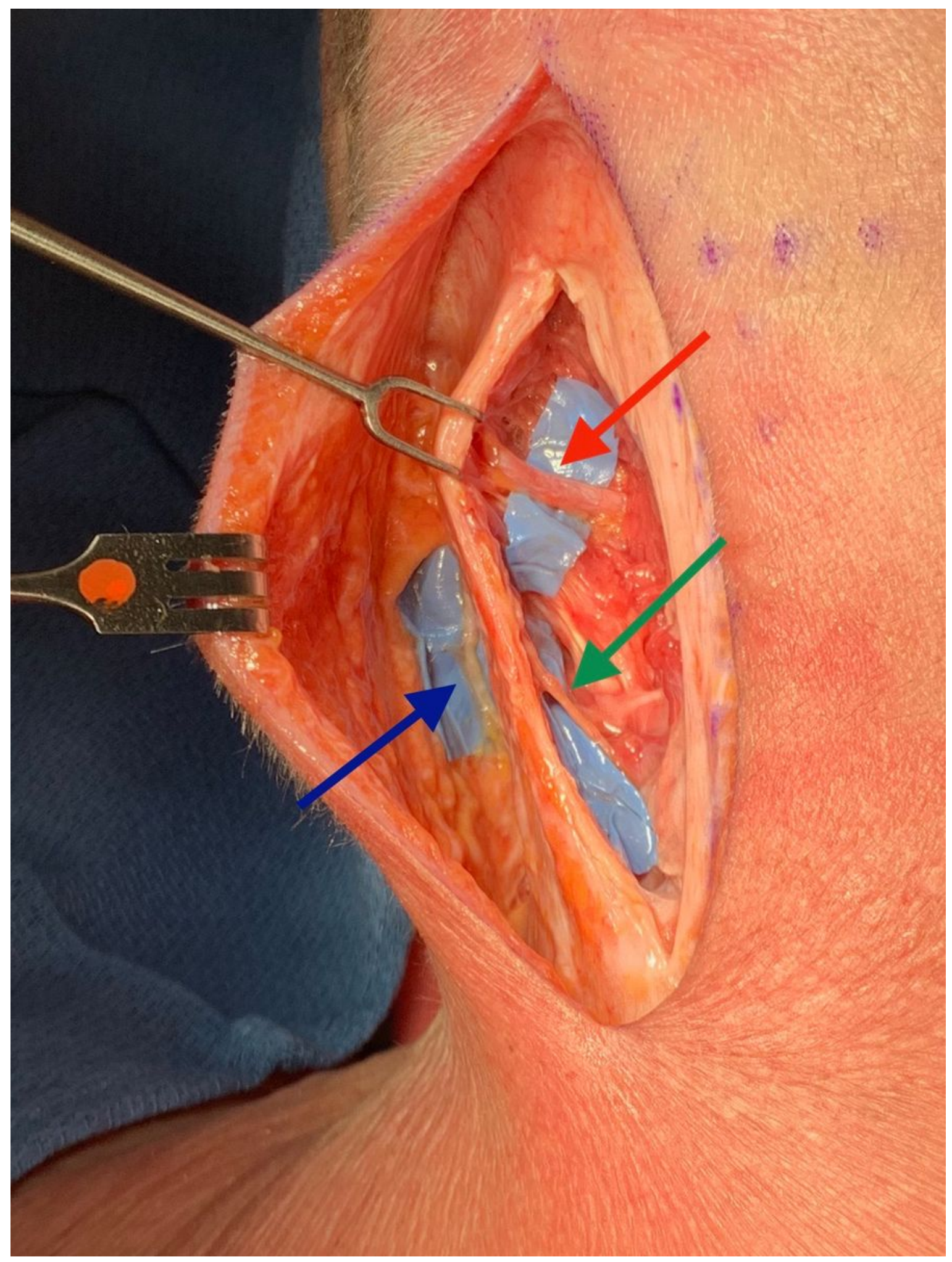

Figure 2

Lateral skin flap raised just above the investing layer of the deep cervical fascia with exposed GON (red arrow), LON (blue arrow), TON (green arrow). 


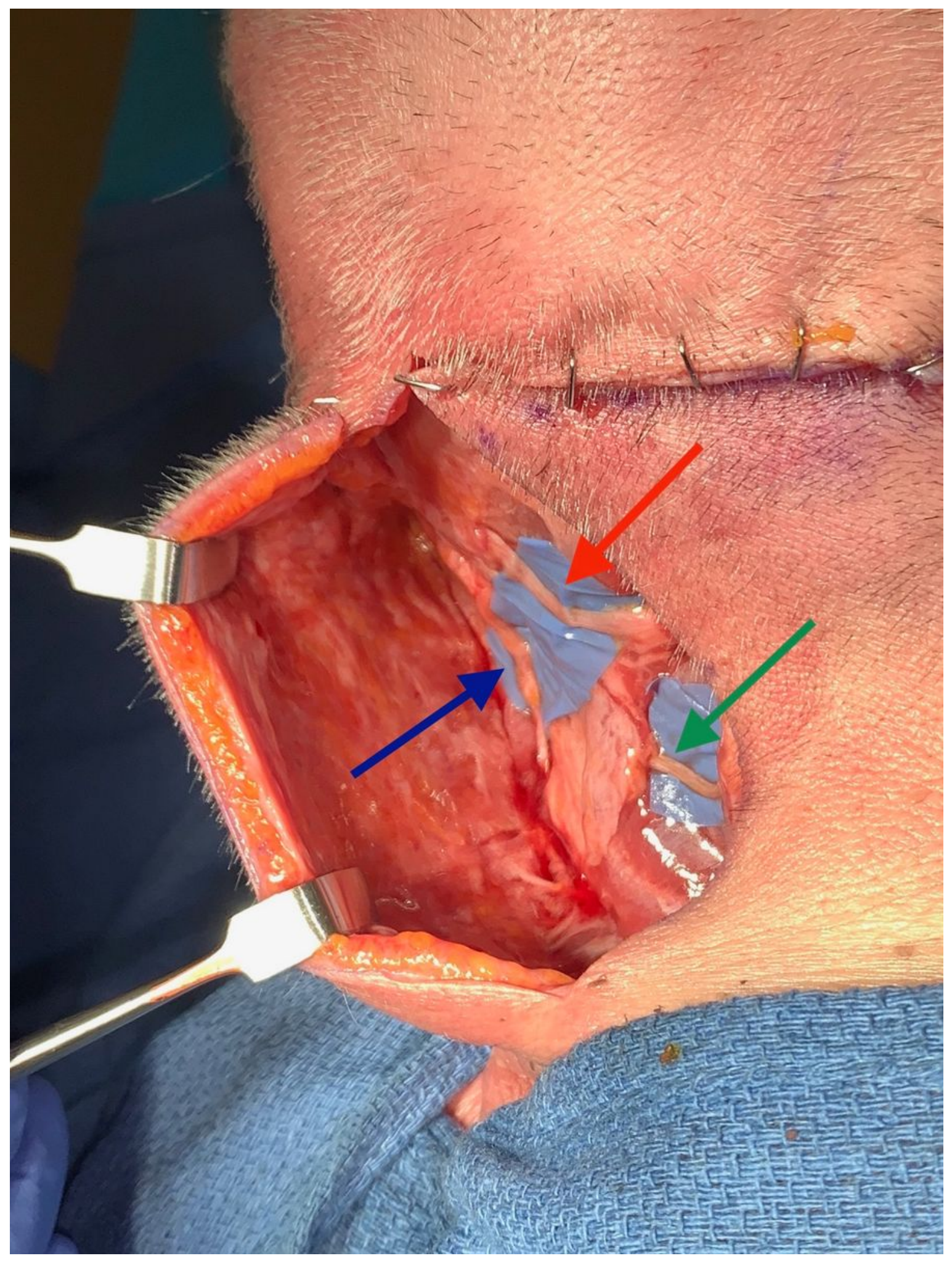

Figure 3

Exposed GON (red arrow), LON (blue arrow), TON (green arrow) through single midline incision. 